\title{
Fracture toughness modelling of polymers filled with inhomogeneously distributed rigid spherical particles
}

\author{
B. Lauke* \\ Leibniz-Institut für Polymerforschung Dresden e.V., Hohe Str. 6, 01069 Dresden, Germany
}

Received 25 November 2016; accepted in revised form 8 February 2017

\begin{abstract}
Filler particles are not homogeneously distributed in real composites consisting of polymer matrices and rigid spherical particles. In the presence of a crack, high multiaxial stresses develop in the material which are a function of the distance from the crack tip. This variation effects the stress concentration in the periphery of the inhomogeneously distributed particles. For a certain stress level closer particles are able to debond from the matrix while particle pairs with larger centre to centre distances may still be bonded to the matrix. Based on a two particle model, the debonding and matrix yielding energies were calculated. Subsequent integration over the local composite deformation results in the fracture toughness of such composites. Randomly distributed particles provide a lower fracture toughness in modelling as compared to inhomogeneoulsy distributed particles in the composite.
\end{abstract}

Keywords: polymer composites, fracture toughness, modeling and simulation

\section{Introduction}

Polymer materials filled with particles show a very complex variation of fracture toughness with particle fraction. It increases with increasing particle volume fraction towards a maximum for thermosets, and it might increase or decrease for thermoplastics. Different failure mechanisms are responsible for this behaviour as, for example, particle debonding and brittle or ductile matrix fracture.

Two main groups of filled polymers can be distinguished. On the one hand the tough matrices (for example, polypropylene, polyethylene) filled with a rigid second phase (e.g. glass, calcium carbonate, mica). And, on the other hand, brittle polymers (e.g. polymethylmethacrylate, polystyrene, epoxy) filled with a second phase of rubber or rigid fillers. While the first group of materials is mainly directed to improve the stiffness and strength with a reasonable toughness, the second one is developed to get composites with an improved toughness, while rubber particles tend to reduce stiffness. In case of preformed rubber particle-modified brittle polymers, the stiffness might not significantly decrease.

Throughout the last decades, the subject of improving the mechanical properties of particle filled polymers received large attention and a bulk of publications are existing. Surveys about this development were conducted by Moloney et al. [1] and by Fu et al. [2]. Interfacial debonding determines the initiation and development of the damage process. For glass bead filled epoxies, Lee and Yee [3, 4] observed that microcracking is mainly caused by debonding. However, it was observed that it is limited to a region near the fracture surface and does not occur in a large dissipation zone around the propagating crack. Yielding processes, as diffuse shear yielding and micro shear banding, are addressed to be more important for toughening of such composites. Wetzel et al. [5] showed that the particles within epoxy nanocomposites induce various fracture mechanisms as crack 
deflection and plastic deformation. It was shown that nanoparticle composites improve stiffness, strength and fracture toughness at the same time. Scanning electron microscopy of silica nanoparticle-reinforced epoxy polymers revealed that debonding of the nanoparticles and subsequent plastic void growth are responsible for toughness increase, cf. Dittant and Pearson [6] and Liu et al. [7]. A significant improvement of fracture toughness with increasing filler content was found.

Kinloch et al. [8] and Hsieh et al. [9] examined hybrid epoxy composites consisting of rubber microparticles and silica nanoparticles and found a drastic improvement of fracture toughness. Two toughening mechanisms were again seen to be responsible: plastic shear-yield bands and debonding of the silica nanoparticles followed by plastic void growth of the epoxy. The debonding process was modelled by Chen et al. [10] and was extended accounting for the existence of an interphase between particle and matrix by Zappalorto et al. [11]. It is well known that the adjacent polymer chains are disordered due the addition of the filler, leading to the formation of an interphase zones; its importance was discussed recently by Zappalortao et al. [12]. However, this phenomenon is more common in case of nanocomposites and will not be considered herein. The contribution of particle debonding to composite toughness was calculated by Williams [13] and in [14]. And it was shown that it provides only a minor direct contribution to energy dissipation, but triggers subsequent yielding processes.

A profound contribution to the modelling of particle composites was provided by Quaresimin et al. [15]. The model accounts the major contribution to energy dissipation, i.e. particle debonding, plastic voiding and shear banding. The comparison between theory and different experiments showed a rather good agreement, supporting the value of the proposed model.

Recently Hamdia et al. [16] published an evaluation of the performance of different models to predict fracture toughness with respect to experimental data. For this they used the so-called Bayesian methodology which considers the different sources of uncertainties in the different models. A not yet considered characteristic material parameter, i.e. the local inhomogeneous particle distribution (particle density variation), will be considered herein.

During the production process of particle reinforced polymers, the particles are distributed within the matrix. The common aim is to reach a homogeneous distribution without agglomeration of particles. However, this is practically not reached. Especially in nano-particle reinforced elastomers, aggregates and agglomerates determine the microstructure of the composites, see for example Morozov et al. [17].

The present work is concerned with the modelling of fracture toughness of polymers filled with rigid fillers which show particle agglomeration during processing of the composites. The basis of the modelling is micromechanics and statistics of particle arrangements. The aim is to provide a model of fracture toughness calculation based on an analytical consideration of the main failure mechanisms, as debonding and local plastic yielding within a dissipation zone and matrix failure in the process zone immediately in front of the crack tip. A main issue of the modelling is that the particles are not homogeneously distributed and this is a new point not yet considered in the literature according to the author's knowledge.

It is assumed that there certain regions with higher particle densities compared to the mean density exist. This leads to overloaded gaps between close particles where debonding may be initiated and which is succeeded by plastic voiding of the matrix material.

It is known from linear elastic fracture mechanics that the stress state in front of a crack is multiaxial and depends on the distance from the crack tip as well as on the direction. Herein this stress field is reduced to a uniaxial one and the concrete shape of the dissipation zone is only parametrically considered, and their change during crack growth was neglected because it is of second order. Next step is the calculation of the specific debonding and yielding energy densities of all particles within the dissipation zone as a function of the applied load. The fracture toughness of the composite is obtained by the integration of the mechanisms over the local composite deformation, because it is highest near the crack tip and decreases towards the dissipation zone edge. Summing up, these dissipation energies with the crack resistance of the matrix met by the propagating crack provide eventually the composite fracture toughness.

\section{Micromechanical model of particle-particle interaction, probability of debonding}

Incorporation of particles into a matrix causes stress concentrations in the periphery of the particle when 
the composite is under loading. Stress fields between particles were calculated by the finite-element method, for example by Böhm et al. [18] and Tzika et al. [19] with the quoted literature in these papers. But, there are also analytical models for the two-particle problem, as for example by Wang et al. [20]. The two-particle arrangement is the typical geometry that should allow the description of the main features of the mechanical problem. For the calculation of the local stress and strain situation around neighbouring particles and the probability of debonding, a basic idea proposed by Weiss [21] was used.

Two rigid particles with diameter, $d$, within an infinite matrix are considered as the representative element, shown in Figure 1. The axis between the centres of the particles has an angle of $\theta$ to the applied deformation, $\varepsilon$, and the centres are in a distance, $r$. Loading of this element leads to deformations in the gap between the particles. When this deformation reaches a critical value, $\varepsilon_{\mathrm{d}}$, debonding starts at one of the particles. It is assumed herein that the initiated debonding crack extends all around this particle at this applied load. The debonding may cause, subsequently, plastic matrix voiding in a matrix shell surrounding the particle.

To simplify modelling, the multiaxial deformation state in front of the crack is approximated by the most important contribution in the direction of loading, $\varepsilon$ (mode one loading). In the crack plane directions, i.e. perpendicular to the applied load, the deformations are neglected. Consequently, the strain

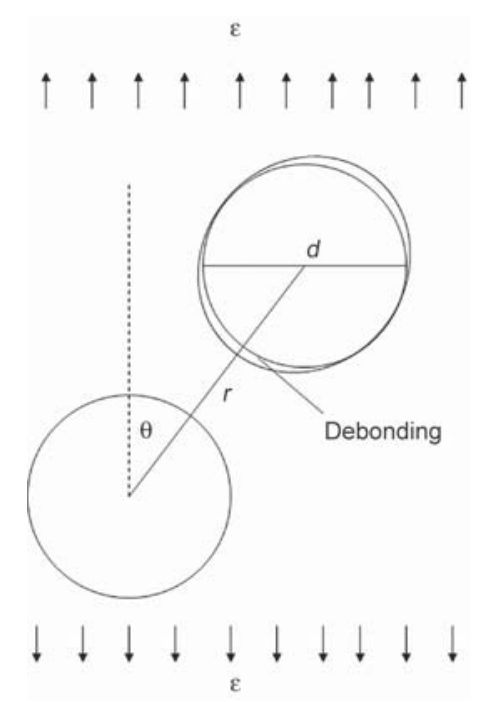

Figure 1. Representative element for the analytical modelling of local deformation; $d$ : particle diameter, $r$ : centre to centre distance, $\varepsilon$ : applied strain causing debonding of one particle in a particle pair tensor reduces to a scalar. Under the applied deformation, $\varepsilon$, the displacement in the matrix is given by $u_{\mathrm{m}} \approx \varepsilon \cdot r \cos \theta$, where the Poisson's effect is neglected and particles are assumed to be rigid. On the other hand, the matrix deformation in the gap is given by: $\varepsilon_{\mathrm{m}} \approx u_{\mathrm{m}} /(r-d)$ providing finally (Equation (1)):

$$
\varepsilon_{\mathrm{m}} \approx \varepsilon \frac{r}{r-d} \cos \theta=\varepsilon \frac{\bar{r}}{\bar{r}-1} \cos \theta:=\varepsilon \alpha \cos \theta
$$

with the normalization $\bar{r}=r / d$ and strain concentration factor: $\alpha=\bar{r} /(\bar{r}-1)$.

The maximum loading is obtained when the particleparticle axis lies in the direction of loading, $\theta=0$. As can be seen in Equation (1), the strain concentration becomes highest when particles are very close to each other. When the matrix deformation reaches a critical value, the debonding process starts at $\varepsilon_{\mathrm{m}}=\varepsilon_{\mathrm{d}}$, with $\varepsilon_{\mathrm{d}}$ as the debonding strain.

Any arbitrary chosen particle of statistically distributed particles has a surrounding, which is characterised by the fact that it debonds as soon as a neighbouring particle is located within this region. This region is limited by $\varepsilon_{\mathrm{m}} \geq \varepsilon_{\mathrm{d}}$ leading with Equation (1) to (Equation (2)):

$$
\frac{1}{\bar{\varepsilon}}<\alpha \cos \theta
$$

with $\bar{\varepsilon}=\varepsilon / \varepsilon_{\mathrm{d}}$.

The maximum radial extension of this region is given in the direction of $\theta=0$ with Equation (2) by Equation (3):

$\overline{r_{\max }}=\frac{1}{1-\bar{\varepsilon}}, \quad \bar{\varepsilon}<1$

The Equations (1)-(3) describe the local deformation situation around one particle under uniaxial loading $\varepsilon$.

To understand the local behaviour around the crack tip, an interesting question is: What is the mean number of neighbouring particles, $N(\varepsilon)$, for a certain applied deformation, $\varepsilon$, which can cause debonding. An answer can be given by using the basics of statistical mechanics as provided, for example by Ziman [22]. The mean number of neighbouring particles within the volume, $V_{\mathrm{d}}$, where debonding can take place, is characterised by the integral of the pair distribution function, $g(r)$, over this volume as shown by Equation (4):

$N=\bar{n} \int_{\mathrm{V}_{\mathrm{d}}} g(r) \mathrm{d} V=\bar{n} \int_{\mathrm{V}_{\mathrm{d}}} g(r) r^{2} \sin \theta \mathrm{d} r \mathrm{~d} \theta \mathrm{d} \phi$ 
where $\bar{n}=6 v /\left(\pi \mathrm{d}^{3}\right)$ is the mean particle density, with $v$ as the particle volume fraction and $g(r)$ as the ratio between the local particle density and the mean particle density, $\bar{n}$.

Because the distribution function $g(r)$ is only a function of the particle-particle distance, $r$, the integration over the angles can be carried out. The circumferential angle, $\phi$, provides $2 \pi$ and during integration over the angel $\theta$ it must be considered that it is limited by $\cos \theta_{\max }=(1 / \bar{\varepsilon})(\bar{r}-1) / \bar{r}$ because of Equation (2).

Finally, the following Equation (5) for the mean number of particles which can cause debonding at a certain applied deformation is obtained as a function of particle volume fraction and local composite deformation, $\bar{\varepsilon}$ :

$N(\bar{\varepsilon}, v)=12 v \int_{1}^{\bar{r}_{\max }} \bar{r}^{2} g(\bar{r})\left(1-\frac{1}{\bar{\varepsilon}} \frac{\bar{r}-1}{\bar{r}}\right) \mathrm{d} \bar{r}$

Considering the injection moulding process during composite manufacturing, it cannot be expected that the particle distribution is homogeneous. For this situation exists a reasonable distribution function, namely that of the hard-core gas, which considers a higher frequency of closely spaced particles. It is one of the simplest functions and is used herein to demonstrate the course of modelling, but it is not a limitation of generality because any other, if experiments are available, more realistic function might be used instead. In reality, with a filled polymer this frequency should be even higher as this one, because very often agglomeration takes place. This distribution function $\bar{r} g(\bar{r})$ as given by Rice and Gray [23] was approximated by a polynomial obtained from a regression analysis (Equation (6)):

$$
\bar{r}^{2} g(\bar{r})=(1+8 v) \bar{r}-6 v \bar{r}^{3}+\frac{v}{2} \bar{r}^{5}
$$

Inserting this expression into Equation (5) provides the mean particle number $N(\bar{\varepsilon}, v)$ in the periphery of one particle which may cause debonding Equation (7):

$$
\begin{aligned}
N(\bar{\varepsilon}, v) & =\frac{12 v}{\bar{\varepsilon}}\left[\frac{1}{6}(1-\bar{\varepsilon})^{-2}-\frac{\bar{\varepsilon}}{3}-\frac{1}{6}\right]+ \\
& +\frac{12 v^{2}}{\bar{\varepsilon}}\left[\frac{1}{60}(1-\bar{\varepsilon})^{-5}-\frac{1}{2}(1-\bar{\varepsilon})^{-3}+\right. \\
& \left.+\frac{4}{3}(1-\bar{\varepsilon})^{-2}-\frac{5}{4} \bar{\varepsilon}-\frac{51}{60}\right]
\end{aligned}
$$

For carbon black filled rubber, the pair distribution functions were recently determined by Morozov et al. [24] for different filler contents; such curves could be alternatively used instead of Equation (6) without changing the general derivations.

In case of a random particle distribution, the pair distribution function would be $g(r)=1$, i.e. the local particle density is equal to the mean value. In this case, the above equation for $N$ is changing into Equation (8):

$N(\bar{\varepsilon}, v)=\frac{2 v}{\bar{\varepsilon}}\left[(1-\bar{\varepsilon})^{-2}-1-2 \bar{\varepsilon}\right]$

The characteristic volume, $V_{\mathrm{d}}$, is that volume within which the local strain concentrations are high enough to cause debonding at the particle/matrix interface. The Poisson distribution is used to calculate the probability, $P(x)$, to find a certain number, $x$, of particles in the considered characteristic volume: $P(x)=$ $N^{\mathrm{x}} / x ! \cdot \exp (-N)$ where $N$ gives the mean number of particles in that volume. The probability to find no particle $(x=0)$ in the volume is: $P(0)=\exp (-N)$ and the probability to find at least one particle is: $P(x \geq 1)=$ $1-\exp (-N)$. If, additionally, the fact is considered that only one particle of a pair is able to debond from the matrix, then the debonding probability of the considered particle is given by Equation (9):

$P(x \geq 1)=1-\exp \left(-\frac{N}{2}\right)$

The knowledge of this probability provides one basis for the calculation of dissipation energy densities for debonding and subsequent matrix yielding.

\section{Fracture toughness}

\subsection{Basic model for calculation of fracture toughness (crack resistance)}

In the following the energy concept is used. To propagate a crack, enough energy must be available: the energy release rate (available from the change of the elastic energy and the applied load) that is at least equal to the energy necessary to initiate the crack propagation. This is expressed usually as sown in Equation (10):

$G \geq G_{\mathrm{c}}=R$

This is a necessary condition; to move the crack, the changes of the energies of both sides must be considered, i.e. the derivatives to the crack length. However, in the following, only crack initiation is considered. This means that only the critical value (fracture toughness) of $R=G_{\mathrm{c}}$ must be determined and not the whole function for crack growth must be 


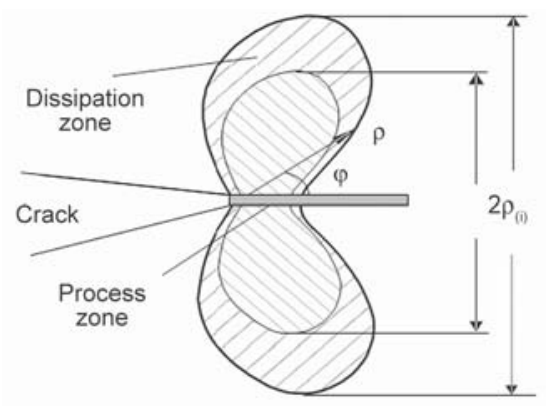

Figure 2. Process and dissipation zones in front of the crack, $\rho, \varphi$ polar coordinates, $\rho_{(\mathrm{i})}$ are the radii of the matrix yielding and debonding zone, respectively

calculated. In order to balance the effect of different structural changes on the crack resistance, it is necessary to consider the processes near the crack tip in more detail. The fracture processes act in different zones, on the one hand there are processes immediately near the crack surfaces, which is termed as process zone. The second group are the more extended structural processes, which take place in the socalled dissipation zone. These zones are given in Figure 2 with the different mechanisms ( $i$ ). This kind of subdivision of the region in front of the crack can be traced back to the works by Evans and Faber [25]. The total crack resistance can be calculated by the contributions of the process zone energy (index $p z$ ) and the debonding (index $d d$ ) and yielding (index $d y)$ contributions within the dissipation zone, respectively, as given by Equation (11):

$$
R_{\mathrm{c}}=R_{\mathrm{pz}}+R_{\mathrm{dd}}+R_{\mathrm{dy}}=R_{\mathrm{pz}}+2 \sum_{\mathrm{i}} \int_{0}^{\rho_{\mathrm{i}}} \eta_{\mathrm{i}}(\rho) \mathrm{d} \rho
$$

with $\rho_{\mathrm{i}}$ as the maximum distance from the crack tip for the initiation of the dissipation mechanism $(i=$ $d, y) ; \eta_{\mathrm{i}}$ is the volume density of the corresponding dissipated energy. For the calculation of the dissipation zone radii, the approximation for the plastic zone is extended to several energy dissipation mechanisms in the composite Equation (12):

$$
\rho_{\mathrm{i}} \cong \beta R_{\mathrm{c}} E_{\mathrm{c}} \frac{1}{\left(\sigma^{\mathrm{i}}\right)^{2}}=\beta \frac{R_{\mathrm{c}}}{E_{\mathrm{c}}} \frac{1}{\left(\varepsilon^{\mathrm{i}}\right)^{2}}
$$

where $E_{\mathrm{c}}$ is the Young's modulus of the composite, and $\sigma^{\mathrm{i}}$ (with $\sigma^{\mathrm{i}}=E_{\mathrm{c}} \varepsilon^{\mathrm{i}}$ ) is the local stress in the composite in front of the crack when the material instability $(i=d, y)$ initiates; the parameter $\beta$ characterises the shape of the dissipation zone and can be used as a fitting parameter.

Now, it is necessary to consider possible mechanisms in the particle composite. The debonding process is one of the dissipation mechanisms that are initiated between particles within the dissipation zone. After debonding of one particle, plastic voiding of the surrounding matrix may be initiated under the stress/ strain field in front of the crack, which is multiaxial. For the consideration of these mechanisms, appropriate particle distribution functions must be taken into account. A description of the real geometrical situation is obtained by using an appropriate pair distribution function, $g(r)$, characterising the local particle density to the mean particle density as function of the centre-centre distance of two neighbouring particles. It must be bared in mind that particle pairs far away from the crack tip, where the high stresses near the crack tip are already faded away, with a very narrow centre-centre distance, $r$, still develop high strain concentrations and may debond. This can easily be seen in Equation (1); for $\bar{r} \rightarrow 1$ the strain concentration approaches infinity, and this causes debonding or yielding also in regions far away from the crack tip. However, on the other hand, the number of neighbouring particles of a chosen single particle that may cause debonding, i.e. $N(\bar{\varepsilon})$ given by Equation (7) and (8) decreases with decreasing $\bar{\varepsilon}$. This means, that away from the crack there may be particle debonding, but only a relatively small number of neighbouring particles are available to cause debonding, in other words: a lower probability for debonding, $P$, (as seen in Equation (9)) exists.

Much higher debonding probability will be found near the crack surface, where the stresses and strains are higher. This effect was observed and described by Lee and Yee [3, 4].

The dissipation processes are shown in Figure 3. After debonding, a matrix yield zone spreads over a certain volume around the debonded particle. The shape and size depends on the stresses and strains in that volume. To simplify the calculations, the following approximation was used. For the debonding and

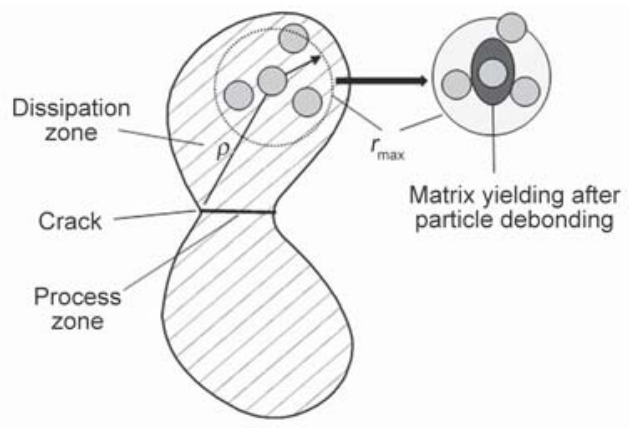

Figure 3. Cross sectional view of the dissipation processes in front of the crack 
yielding processes only the strain component, $\varepsilon$, in the direction of the applied mode I loading direction was considered for the particle pair interaction, see Figure 1. After debonding near the particle pole, the debonding crack propagates all around the particle. The subsequent yielding process within the matrix shell was also considered under such loading.

The necessary integration over the distance, $\rho$, from the crack plane in Equation (11) can be transformed into integration over the deformation, $\varepsilon$, which is more appropriate in this case, because the specific dissipation energy can be derived as a function of the applied strain.

With the replacement: $\mathrm{d} \rho=-2 \beta\left(R_{\mathrm{d}} / E_{\mathrm{c}}\right)(\varepsilon)^{-3} \mathrm{~d} \varepsilon$ the integral in Equation (11) can be written as Equation (13):

$R_{\mathrm{dd}}=2 \int_{0}^{\rho_{\mathrm{d}}} \eta_{\mathrm{d}}(\rho) \mathrm{d} \rho=4 \beta \frac{R_{\mathrm{c}}}{E_{\mathrm{c}}} \int_{\varepsilon_{0}}^{\varepsilon_{\mathrm{d}}} \eta_{\mathrm{d}}(\varepsilon) \frac{1}{\varepsilon^{3}} \mathrm{~d} \varepsilon$

The integration limit of $\rho_{\mathrm{d}}$ beyond which no debonding can be initiated, was replaced by the deformation, $\varepsilon=\varepsilon_{0}$, which corresponds to the applied mean deformation of the specimen. For the determination of the smallest value of deformation where debonding starts, the following must be considered: For particle pairs that nearly touch each other, i.e. $\bar{r} \rightarrow 1$, already at low deformations close particle pairs can debond. The local strain, $\varepsilon$, in front of the crack reaches the mean value of the composite strain, $\varepsilon_{0}$, away from the crack tip. On the other hand, near the crack tip $(\rho \rightarrow 0)$, the stresses and strains show extreme high values. This means that even particle pairs with largest distances may debond for the highest local strain $\varepsilon \rightarrow \varepsilon_{\mathrm{d}}$.

Rearranging Equation (11) provides for the composite crack resistance if only the debonding mechanism is considered (Equation (14)):

$$
R_{\mathrm{c}}=\frac{R_{\mathrm{pz}}}{1-\frac{4 \beta}{E_{\mathrm{c}}} \int_{\varepsilon_{0}}^{\varepsilon_{\mathrm{d}}} \eta_{\mathrm{d}}(\varepsilon) \frac{1}{\varepsilon^{3}} \mathrm{~d} \varepsilon}
$$

After particle debonding, the matrix region around this particle may start yielding (plastic voiding). Integration of the deformation field in front of the crack over the debonding zone allows to calculate this contribution to the dissipation zone energy (Equation (15)):

$$
R_{\mathrm{dy}}=2 \int_{0}^{\rho_{\mathrm{y}}} \eta_{\mathrm{y}}(\rho) \mathrm{d} \rho=4 \beta \frac{R_{\mathrm{c}}}{E_{\mathrm{c}}} \int_{\varepsilon_{0}}^{\varepsilon_{\mathrm{d}}} \eta_{\mathrm{y}}(\varepsilon) \frac{1}{\varepsilon^{3}} \mathrm{~d} \varepsilon
$$

Inserting Equations (13) and (15) into Equation (11) provides the crack resistance of the composite, given by Equation (16):

$$
R_{\mathrm{c}}=\frac{R_{\mathrm{pz}}}{1-\frac{4 \beta}{E_{\mathrm{c}}}\left(\int_{\varepsilon_{0}}^{\varepsilon_{d}}\left(\eta_{d}(\varepsilon)+\eta_{y}(\varepsilon)\right) \frac{1}{\varepsilon^{3}} d \varepsilon\right.}
$$

\subsection{Calculation of dissipation energies and fracture toughness}

Now, the specific energies for debonding and yielding will be derived. As shown in section 2, the probability for the debonding of a considered particle of the composite is equal to $P(N(\bar{\varepsilon}))$ and is a function of the number of particles in the periphery which can cause debonding. The local situation in the periphery of one particle is shown in Figure 3.

The volume specific debonding energy, $\eta_{\mathrm{d}}$, can now be calculated by multiplying the debonding energy of one particle, $W_{\mathrm{d}}=G_{\mathrm{d}} \pi d^{2}$, by the probability of debonding, $P$, and normalized by $V\left(r_{\max }\right)=4 \pi r_{\max }^{3} / 3$ as the volume within which the debonding occurs, i.e. it is limited by the maximum possible neighbouring particle distance, $r_{\max }$. This leads to the volume specific debonding energy given by Equation (17):

$\eta_{\mathrm{d}}=\frac{G_{\mathrm{d}} \pi d^{2}}{V\left(r_{\max }\right)} P$

with $G_{\mathrm{d}}$ as the specific debonding energy between particle and matrix. Using Equation (3) provides the dependence of this energy on the applied deformation in front of the crack (Equation (18)):

$$
\eta_{\mathrm{d}}(\bar{\varepsilon})=\frac{3}{4} \frac{G_{\mathrm{d}}}{d}(1-\bar{\varepsilon})^{3} P(N(\bar{\varepsilon}))
$$

where $P$ is given in Equation (9) and $N$ in Equations (7) or Equation (8), respectively.

Nicholson [26] and Chen et al .[10] considered theoretically the problem of particle debonding from a surrounding matrix. The debonding strength, $\sigma_{\mathrm{d}}$, depends on the particle size and on the specific debonding energy, $G_{\mathrm{d}}$, at the particle/matrix interface (Equation (19)):

$\sigma_{\mathrm{d}}=\varepsilon_{\mathrm{d}} E_{\mathrm{m}}=\sqrt{\frac{8 G_{\mathrm{d}}}{d} \frac{E_{\mathrm{m}}}{\left(1+v_{m}\right)}}$

By inserting this relation into Equation (18), one gets finally for the debonding energy density (Equation (20)): 
$\eta_{\mathrm{d}}(\bar{\varepsilon})=\frac{3}{32}\left(1+v_{\mathrm{m}}\right) E_{\mathrm{m}} \varepsilon_{\mathrm{d}}^{2}(1-\bar{\varepsilon})^{3} P(N(\bar{\varepsilon}))$

It is interesting to note that this energy density is independent of the particle diameter, similar to the outcome of the previous modelling, cf. [27], if the validity of Equation (19) is supposed.

After debonding of the particle, the matrix shell around it starts plastic voiding, cf. Figure 3. This process was discussed in detail by Williams [13] and in Ref. [14]. Herein a slightly simpler approximation is used, however, the result of Ref. [14] for the yielding energy of one matrix shell could also be used herein.

The energy for plastic matrix voiding was calculated by the product of the volume specific energy, $\omega_{\mathrm{m}}$, with the relevant matrix volume fraction, $v_{\mathrm{m}}$, and the probability that that process starts, which is equal to the probability of particle debonding, $P: \eta_{\mathrm{y}}=\omega_{\mathrm{m}} v_{\mathrm{m}} P$. On the other hand, the matrix volume decreases with the number of particles in the neighbourhood, because these particles limit the extension of the voiding process. An approximation is given by Equation (21):

$$
\eta_{y}=\omega_{m} v_{m} P=\omega_{m}(1-P(N(\bar{\varepsilon})))(1-v) P(N(\bar{\varepsilon}))
$$

where $v$ is the particle volume fraction. The volume specific voiding energy, $\omega_{\mathrm{m}}$, is used here as a material parameter, which in reality is a function of particle size. It was calculated in paper [14] for a mean particle size and in paper [28] for particle size distributions. In this simple approximation as a parameter, the particle size dependence cannot be described, however, it can be done if the equations of the previous papers are used instead.

The process zone energy is determined by the fracture of deformed matrix material; the crack meets areas of yielded material with the volume fraction $(1-v)$, Equation (22):

$$
R_{\mathrm{pz}}=R_{\mathrm{m}}(1-v)
$$

Summarizing these considerations, the fracture toughness can be calculated with Equations (16), (20)-(22) by Equation (23):
Herein the composite strain was normalized by the debonding strain: $\bar{\varepsilon}=\varepsilon / \varepsilon_{\mathrm{d}}$ and the normalization was used, with $\varepsilon_{0} / \varepsilon_{\mathrm{d}}=\delta$ as a small number to avoid an infinitesimal maximum debonding radius, $r_{\max }$.

\section{Results and discussion}

\section{Implementation of the model}

The proposed general model was applied for a special system consisting of silica $\left(\mathrm{SiO}_{2}\right)$ particles within epoxy resin. For the matrix material, the following properties were used:

$E_{\mathrm{m}}=3 \mathrm{GPa}, v_{\mathrm{m}}=0.35$ and a debonding strain of $\varepsilon_{\mathrm{d}}=$ 0.1 . The silica spheres have the following material properties: elastic modulus $E_{\mathrm{p}}=64 \mathrm{GPa}$ and Poisson's ratio $v_{\mathrm{p}}=0.2$. The volume specific energy for plastic deformation, $\omega_{\mathrm{m}}$, was used as a parameter, that can be determined by the stress-strain response of the matrix material. For the composite modulus, the relation:

$E_{\mathrm{c}}=E_{\mathrm{m}}[(7+8 v)(2+v)] /[2(1-v)(7+4 v)]$ proposed by Hashin and Shtrikman [29] was applied. And for the $\beta$ parameter the value $\beta=1 / 60$ was used.

The first aim was the calculation of the mean number, $N(\bar{\varepsilon}, v)$, of particles that may cause particle debonding. This generated the basis for calculating the probability for particle debonding, $P$, and the debonding and yielding energies around one particle. The variation of $N$ with the normalized local deformation in front of the crack is shown in Figure 4 for two particle volume fractions. Two different local particle distributions are compared: homogeneously distributed particles with $g(r)=1$ and that of the pair distribution as given in Equation (6). The number of particles in the periphery of one chosen particle is higher for the inhomogeneously distributed particles and becomes larger for higher particle volume fractions, of course. The number $N$ drastically increases for higher local strains, $\bar{\varepsilon}=\varepsilon / \varepsilon_{\mathrm{d}}$, of about $70 \%$, bearing in mind that this may also be considered as the limit for the particle-particle interaction model as given in section 2 .

The normalized fracture toughness, $R_{\mathrm{c}} / R_{\mathrm{m}}$, where $R_{\mathrm{m}}$ is the matrix crack resistance, is shown in Figure 5 for two different volume specific matrix yielding energies, $\omega_{\mathrm{m}}$. This value has a significant influence on

$$
R_{\mathrm{c}}=\frac{R_{\mathrm{m}}(1-v)}{1-\frac{3 \beta E_{\mathrm{m}}\left(1+v_{\mathrm{m}}\right)}{8 E_{\mathrm{c}}}\left[\int_{\delta}^{1} P(N(\bar{\varepsilon}))\left(\frac{1-\bar{\varepsilon}}{\bar{\varepsilon}}\right)^{3} \mathrm{~d} \bar{\varepsilon}\right]-\frac{4 \beta}{\varepsilon_{\mathrm{d}}^{2} E_{\mathrm{c}}} \omega_{\mathrm{m}}(1-v) \int_{\delta}^{1}\left(1-P(N(\bar{\varepsilon})) P(N(\bar{\varepsilon})) \frac{1}{\bar{\varepsilon}^{3}} \mathrm{~d} \bar{\varepsilon}\right)}
$$




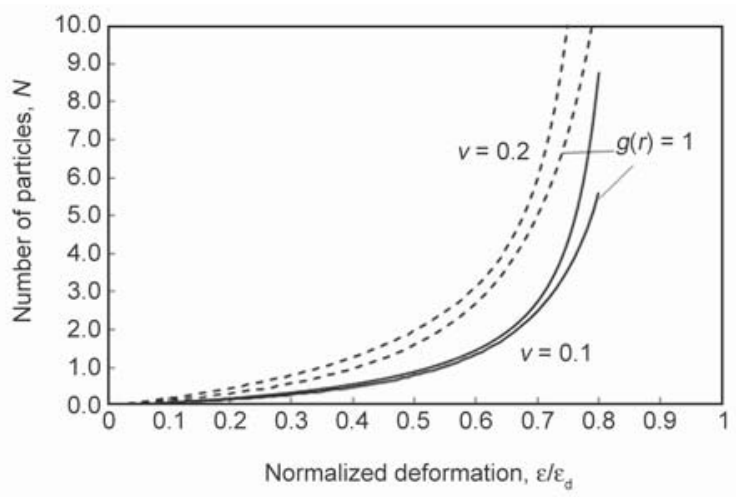

Figure 4. Mean number of neighbouring particles as a function of applied deformation for the random particle distribution with $g(r)=1$ and distribution according to the pair distribution at various particle fractions, $v$

the variation. It becomes clear that the composite fracture toughness increases with increasing particle volume fraction. Such behaviour was measured for epoxy composites filled with nano-silica particles by Tang et al. [30]. They gave for their composite the following values for different silica volume fractions: $v=0, R_{\mathrm{d}} / R_{\mathrm{m}}=1 ; v=0.03, R_{\mathrm{d}} / R_{\mathrm{m}}=1.94 ; v=0.06$, $R_{\mathrm{c}} / R_{\mathrm{m}}=2.27 ; v=0.09, R_{\mathrm{d}} / R_{\mathrm{m}}=2.49 ; v=0.12, R_{\mathrm{c}} / R_{\mathrm{m}}=$ 2.85 . The interesting point is that the model can describe such a variation with $\mathrm{v}$ as shown in Figure 5 within the same quantitative range for a volume specific yielding energy of $\omega_{\mathrm{m}}=100 \mathrm{MPa}$, which was used in the model as a parametric value. An interesting question is which influence has the local particle distribution on fracture toughness; it becomes clear in Figure 6. Surprisingly, toughness is higher for the inhomogeneoulsy distributed particles. Bittmann et al. [31] reported that in nano-composites toughness can be increased when the fillers become more dispersed, i.e. if agglomeration can be avoided. But, agglomerated particles are not the same as some local

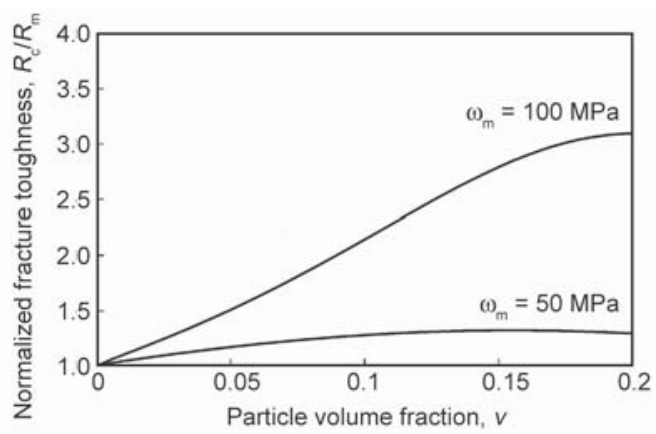

Figure 5. Normalized crack resistance of composites with particle debonding and matrix yielding versus particle volume fraction for different volume specific matrix yielding energies, $\omega_{\mathrm{m}}$

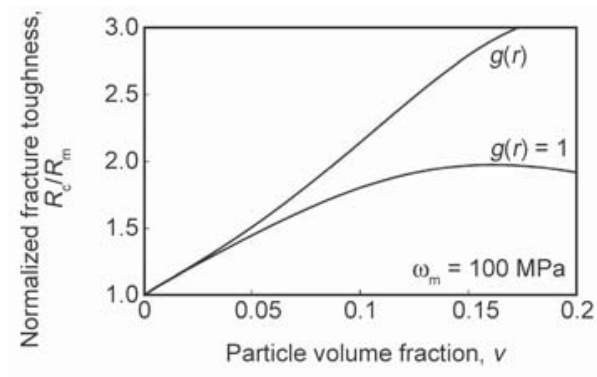

Figure 6. Normalized crack resistance of composites with particle debonding and matrix yielding versus particle volume fraction for the random particle distribution with $g(r)=1$ and distribution according to the pair distribution at various particle fractions, $v$

inhomogeneity in micro-size particle filled composites, where the matrix in between the particles remains the same physical continuum. These differences between nano- and microparticle filled composites were recently discussed by Zidek et al. [32].

\section{Conclusions}

With increasing particle volume fraction, the mean number of the neighbouring particles $N$ increases and, consequently, also the probability of particle debonding increases. This provides by itself an increase in composite toughness, but the absolute value is small, as shown if only the first term of the integral in the denominator of Equation (23) is used.

However, it triggers yielding of the polymer matrix in the periphery. As can be seen in Equation (21), yielding energy density is proportional to the probability for debonding, $P$, and to the factor, $(1-P)$, because the plastic yielding volume decreases accordingly. But, finally, the total composite fracture toughness increases for more inhomogeneously distributed particles. Consequently, inhomogeneously distributed micro-size fillers within polymer matrix seem to have a positive effect on fracture toughness. Unfortunately, the author could not yet find any experimental verification for this geometrical effect in micro-size particle filled polymers in literature. One must bear in mind that most of the available experimental results are for real composites which are expected not to have a random particle arrangement.

\section{References}

[1] Moloney A. C., Kausch H. H., Kaiser T., Beer H. R.: Parameters determining the strength and toughness of particulate filled epoxide resins. Journal of Materials Science, 22, 381-393 (1987).

https://doi.org/10.1007/BF01160743 
[2] Fu S-Y., Feng X-Q., Lauke B., Mai Y-W.: Effects of particle size, particle/matrix interface adhesion and particle loading on mechanical properties of particulate-polymer composites. Composites Part B: Engineering, 39, 933-961 (2008).

https://doi.org/10.1016/j.compositesb.2008.01.002

[3] Lee J., Yee A. F.: Inorganic particle toughening I: Micromechanical deformations in the fracture of glass bead filled epoxies. Polymer, 42, 577-588 (2001). https://doi.org/10.1016/S0032-3861(00)00397-9

[4] Lee J., Yee A. F.: Inorganic particle toughening II: Toughening mechanisms of glass bead filled epoxies. Polymer, 42, 589-597 (2001).

https://doi.org/10.1016/S0032-3861(00)00398-0

[5] Wetzel B., Rosso P., Haupert F., Friedrich K.: Epoxy nanocomposites - Fracture and toughening mechanisms. Engineering Fracture Mechanics, 73, 2375-2398 (2006). https://doi.org/10.1016/j.engfracmech.2006.05.018

[6] Dittanet P., Pearson R. A.: Effect of silica nanoparticle size on toughening mechanisms of filled epoxy. Polymer, 53, 1890-1905 (2012).

https://doi.org/10.1016/j.polymer.2012.02.052

[7] Liu H-Y., Wang G-T., Mai Y-W., Zeng Y.: On fracture toughness of nano-particle modified epoxy. Composites Part B: Engineering, 42, 2170-2175 (2011).

https://doi.org/10.1016/j.compositesb.2011.05.014

[8] Kinloch A. J., Mohammed R. D., Taylor A. C., Eger C., Sprenger S., Egan D.: The effect of silica nano particles and rubber particles on the toughness of multiphase thermosetting epoxy polymers. Journal of Materials Science, 40, 5083-5086 (2005).

https://doi.org/10.1007/s10853-005-1716-2

[9] Hsieh T. H., Kinloch A. J., Masania K., Sohn Lee J., Taylor A. C., Sprenger S.: The toughness of epoxy polymers and fibre composites modified with rubber microparticles and silica nanoparticles. Journal of Materials Science, 45, 1193-1210 (2010).

https://doi.org/10.1007/s10853-009-4064-9

[10] Chen J-K, Wang G-T., Yu Z-Z., Huang Z., Mai Y-W.: Critical particle size for interfacial debonding in polymer/nanoparticle composites. Composites Science and Technology, 70, 861-872 (2010).

https://doi.org/10.1016/j.compscitech.2010.02.004

[11] Zappalorto M., Salviato M., Quaresimin M.: Influence of the interphase zone on the nanoparticle debonding stress. Composites Science and Technology, 72, 49-55 (2011).

https://doi.org/10.1016/j.compscitech.2011.09.016

[12] Zappalorto M., Pontefisso A., Fabrizi A., Quaresimin M.: Mechanical behaviour of epoxy/silica nanocomposites: Experiments and modelling. Composites Part A: Applied Science and Manufacturing, 72, 58-64 (2015). https://doi.org/10.1016/j.compositesa.2015.01.027

[13] Williams G.: Particle toughening of polymers by plastic void growth. Composites Science and Technology, 70, 885-891 (2010).

https://doi.org/10.1016/j.compscitech.2009.12.024
[14] Lauke B.: Contribution of matrix yielding energy to the crack resistance of particle reinforced composites. Composites Science and Technology, 86, 135-141 (2013). https://doi.org/10.1016/j.compscitech.2013.07.004

[15] Quaresimin M., Salviato M., Zappalorto M.: A multiscale and multi-mechanism approach for the fracture toughness assessment of polymer nanocomposites. Composites Science and Technology, 91, 16-21 (2014).

https://doi.org/10.1016/j.compscitech.2013.11.015

[16] Hamdia K. M., Zhuang X., He P., Rabczuk T.: Fracture toughness of polymeric particle nanocomposites: Evaluation of models performance using Bayesian method. Composites Science and Technology, 126, 122-129 (2016).

https://doi.org/10.1016/j.compscitech.2016.02.012

[17] Morozov I., Lauke B., Heinrich G.: A new structural model of carbon black framework in rubbers. Computational Materials Science, 47, 817-825 (2010).

https://doi.org/10.1016/j.commatsci.2009.11.009

[18] Böhm H. J., Eckschlager A., Han W.: Multi-inclusion unit cell models for metal matrix composites with randomly oriented discontinuous reinforcement. Computational Materials Science, 25, 42-53 (2002).

https://doi.org/10.1016/s0927-0256(02)00248-3

[19] Tzika P. A., Boyce M. C., Parks D. M.: Micromechanics of deformation in particle-toughened polyamides. Journal of the Mechanics and Physics of Solids, 48, 18931929 (2000).

https://doi.org/10.1016/S0022-5096(99)00096-4

[20] Wang Z., Zhang J., Guo X.: Two circular inclusions with inhomogeneously imperfect interfaces in plane elasticity. International Journal of Solids and Structures, 42, 2601-2623 (2005).

https://doi.org/10.1016/j.ijsolstr.2004.10.002

[21] Weiß H. J.: Einige Aspekte des mechanischen Verhaltens gefüllter Polymerer (in German). Plaste und Kautschuk, 24, 684-688 (1977).

[22] Ziman J. M.: Model of disorder. Cambridge University Press, London (1979).

[23] Rice S., Gray P.: The statistical mechanics of simple liquids. Interscience, New York (1965).

[24] Morozov I., Lauke B., Tada T.: Structural modelling and stiffness of filled elastomers. Composites Part B: Engineering, 60, 555-560 (2014).

https://doi.org/10.1016/j.compositesb.2014.01.002

[25] Evans A. G., Faber K. T.: Toughening of ceramics by circumferential microcracking. Journal of the American Ceramic Society, 64, 394-398 (1981).

https://doi.org/10.1111/j.1151-2916.1981.tb09877.x

[26] Nicholson D. W.: On the detachment of a rigid inclusion from an elastic matrix. Journal of Adhesion, 10, 255260 (1979).

https://doi.org/10.1080/00218467908544628

[27] Lauke B.: Effect of particle size distribution on debonding energy and crack resistance of polymer composites. Computational Materials Science, 77, 53-70 (2013). https://doi.org/10.1016/j.commatsci.2013.04.017 
[28] Lauke B.: Effect of particle size distribution on fracture toughness of polymer composites considering plastic void growth after particle debonding. Mechanics Research Communications, 66, 1-6 (2015).

https://doi.org/10.1016/j.mechrescom.2015.02.002

[29] Hashin Z., Shtrikman S.: On some variational principles in anisotropic and nonhomogeneous elasticity. Journal of the Mechanics and Physics of Solids, 10, 335-342 (1962).

https://doi.org/10.1016/0022-5096(62)90004-2

[30] Tang L-C., Zhang H., Sprenger S., Ye L., Zhang Z.: Fracture mechanisms of epoxy-based ternary composites filled with rigid-soft particles. Composites Science and Technology, 72, 558-565 (2012).

https://doi.org/10.1016/j.compscitech.2011.12.015
[31] Bittmann B., Haupert F., Schlarb A. K.: Preparation of $\mathrm{TiO}_{2}$ /epoxy nanocomposites by ultrasonic dispersion and their structure property relationship. Ultrasonics Sonochemistry, 18, 120-126 (2011). https://doi.org/10.1016/j.ultsonch.2010.03.011

[32] Zidek J., Kucera J., Jancar J.: Model of random spatial packing of rigid spheres with controlled macroscopic homogenity. Computers, Materials and Continua, 16, 51-73 (2010).

https://doi.org/10.3970/cmc.2010.016.051 